\title{
Interactive Analysis of TX-1123 with Cyclo-oxygenase: Design of COX2 Selective TX Analogs
}

\author{
KAZUTO OHKURA $^{1 *}$, YOHEI TATEMATSU ${ }^{1}$, YUKI KAWAGUCHI ${ }^{2}$, YOSHIHIRO UTO $^{3}$ and HITOSHI HORI ${ }^{3}$ \\ ${ }^{1}$ Graduate School of Pharmaceutical Sciences, \\ Suzuka University of Medical Science Graduate School, Suzuka, Japan; \\ ${ }^{2}$ Faculty of Pharmaceutical Sciences, Chiba Institute of Science, Choshi, Japan; \\ ${ }^{3}$ Department of Biological Science and Technology, Life System, Institute of Technology and Science, \\ The University of Tokushima Graduate School, Tokushima, Japan
}

\begin{abstract}
Background: To date, two cyclo-oxygenase (COX) isoforms, COX1 and COX2, have been identified. In the present study, the COX-inhibitory activities of TX-1123 derivatives with the 2-hydroxyarylidene-4-cyclopentene-1,3dione structure were examined, and the binding profiles of TX-1123 to COXs were analyzed using docking simulations. Materials and Methods: X-Ray data on COXI [protein data bank $(P D B) I D=1 P G G]$ and $C O X 2(P D B I D=3 L N 1)$ were used for molecular interactive simulations. The interactive profiles of TX-1123 derivatives with COXs were examined using a molecular simulation technique with Molegro Virtual Docker (CLC bio, Aarhus, Denmark). Results: TX-1123 exhibited COXI-inhibitory activity [half-maximal-inhibitory concentration $\left.\left(I C_{50}\right)=1.57 \times 10^{-5} \mathrm{M}\right]$. The COX2 inhibitory activity of TX-1123 was potent $\left(I C_{50}=1.16 \times 10^{-6} \mathrm{M}\right)$, and the ratio of COX1/COX2 inhibition was 13.5.TX-1123 bound to the COX2 molecule, and the oxygen atom of the 4cyclopentene-1,3-dione region of TX-1123 interacted with $\mathrm{Cys}^{26}$ and $\mathrm{Gln}^{447}$ of COX2. Conclusion: The TX-1123binding pocket of COX2 differs from that of the COX2selective celecoxib-binding pocket. TX-1123 exhibited a different COX2-interactive mechanism from that of celecoxib.
\end{abstract}

Non-steroidal anti-inflammatory drugs (NSAIDs) are widely used in clinical practice. The anti-inflammatory effect of NSAIDs are attributable to reductions in the formation of

Correspondence to: Professor Kazuto Ohkura, Graduate School of Pharmaceutical Sciences, Suzuka University of Medical Science Graduate School, 3500-3 Minamitamagaki-cho, Suzuka, Mie 5138670. Japan. Tel: +81 593400611, Fax: +81 593681271, e-mail: kohkura@suzuka-u.ac.jp

Key Words: Protein kinase, kinase inhibitor, cyclo-oxygenase. prostaglandins (PG) $(1,2)$. This ability is associated with the inhibition of cyclo-oxygenase (COX), which converts arachidonic acid to $\mathrm{PGH}_{2}$. To date, two COX isoforms, COX1 and COX2, have been identified. COX1 is expressed constitutively and is the housekeeping enzyme responsible for the physiological activities of PG. COX2 is expressed under inflammatory conditions, and is responsible for pathological PGs that produce pain and fever (3-5). The COX2-selective agent celecoxib is widely used as antiinflammatory agent (6).

We previously designed and synthesized a new tyrphostin AG17 analog. Tyrphostin AG17 was shown to suppress the effects of insulin in rat adipocytes (7). Palumbo et al. demonstrated that AG17 induced apoptosis and inhibited cyclin-dependent kinase 2 activity through a mechanism that purportedly did not involve reductions in cellular ATP level (8). Tyrphostin AG17 has been shown to act as an uncoupler of oxidative phosphorylation in isolated rat liver and heart mitochondria (9). Designed tyrphostin AG17 analogs have a 2-hydroxyarylidene-4-cyclopentene-1,3-dione structure (Figure 1). TX-1123 has been shown to possess useful features (e.g. strong Src kinase inhibitory activity and weak mitochondrial toxicity) (10-11). In the present study, we analyzed the COX-inhibitory activities of TX-1123 derivatives, and examined the binding profiles of TX-1123 to COXs using docking simulations.

\section{Materials and Methods}

Assay for COX inhibition. COX1 and COX2 proteins were isolated from ram seminal vesicles and sheep placenta, and the inhibitory effect of test compounds on these COX proteins were examined as previously described (12). COX1- and COX2-inhibitory effect of celecoxib were examined by $\mathrm{COX} 1$-mediated $\mathrm{PGE}_{2}$ production in human lymphoma cells (half maximal-inhibitory concentration, $\mathrm{IC}_{50}$ ) and $\mathrm{COX} 2$-mediated $\mathrm{PGE}_{2}$ production in human dermal fibroblasts $\left(\mathrm{IC}_{50}\right)$, respectively (13). 
<smiles>[R16]c1cc(C=C2C(=O)C=CC2=O)cc(C(C)(C)C)c1C([R4])(C)C</smiles><smiles>[R4][C@@H](C=C1C(=O)C=CC1=O)c1cc(C)c(O)c(C)c1</smiles><smiles>COc1c(C)cc(C=C2C(=O)C=CC2=O)cc1C(=O)O</smiles>

Figure 1. Structure of TX-1123 derivatives.

Interactive analysis of TX-1123 with COX. The X-ray data sets of COX1 (protein data bank ID=1PGG) and COX2 (ID=3LN1) were used for molecular interactive simulation. The interactive analysis between TX-1123 derivatives and COXs were examined by molecular simulation technique [Molecular Mechanics (MM) Molecular Dynamics (MD)] using InsightII Discover under consistent valence force field (Accelrys Inc., San Diego, CA, USA) and Molegro Virtual Docker (CLC bio, Aarhus, Denmark) as previously described $(11,14-15)$. The ligand-bindable pocket of COXs were examined using Molegro Virtual Docker.

\section{Results}

Effects of TX-1123 derivatives on COX activity. TX-1123 exhibited COX1-inhibitory activity, and its half maximalinhibitory concentration $\left(\mathrm{IC}_{50}\right)$ was $1.57 \times 10^{-5} \mathrm{M}$ (Table I). The COX2-inhibitory activity of TX-1123 was potent $\left(\mathrm{IC}_{50}=1.16 \times 10^{-6} \mathrm{M}\right)$, and the ratio of $\mathrm{COX} 1 / \mathrm{COX} 2$ inhibition $\left(\mathrm{R}_{\mathrm{C} 1 / \mathrm{C} 2}\right)$ was $13.5 . \mathrm{R}_{\mathrm{C} 1 / \mathrm{C} 2}$ is an index of COX2 selectivity. The COX2-inhibitory effect of TX-1123 was higher than that of the COX1-inhibitory effect. TX-1925 exhibited inhibitory activity toward $\mathrm{COX} 1 \quad\left(\mathrm{IC}_{50}=4.77 \times 10^{-5} \mathrm{M}\right)$ and $\mathrm{COX} 2$ $\left(\mathrm{IC}_{50}=1.03 \times 10^{-5} \mathrm{M}\right)$, and the $\mathrm{RC} 1 / \mathrm{C} 2$ was 4.63 . The $\mathrm{R}_{\mathrm{C} 1 / \mathrm{C} 2}$ values of TX-1918, TX-1926, and TX-1934 were less than 1.0 (0.11-0.77), and these TX compounds were not selective for COX2. Tyrphostin AG17 only weakly inhibited COX1 and $\mathrm{COX} 2$, with $\mathrm{IC}_{50}$ values of 10 -fold or greater than those of the TX derivatives.

The COX-inhibitory activity of celecoxib, which is a known COX2-selective drug, was demonstrated using the production of $\mathrm{PGE}_{2}$ in human lymphoma cells, and its $\mathrm{IC}_{50}$

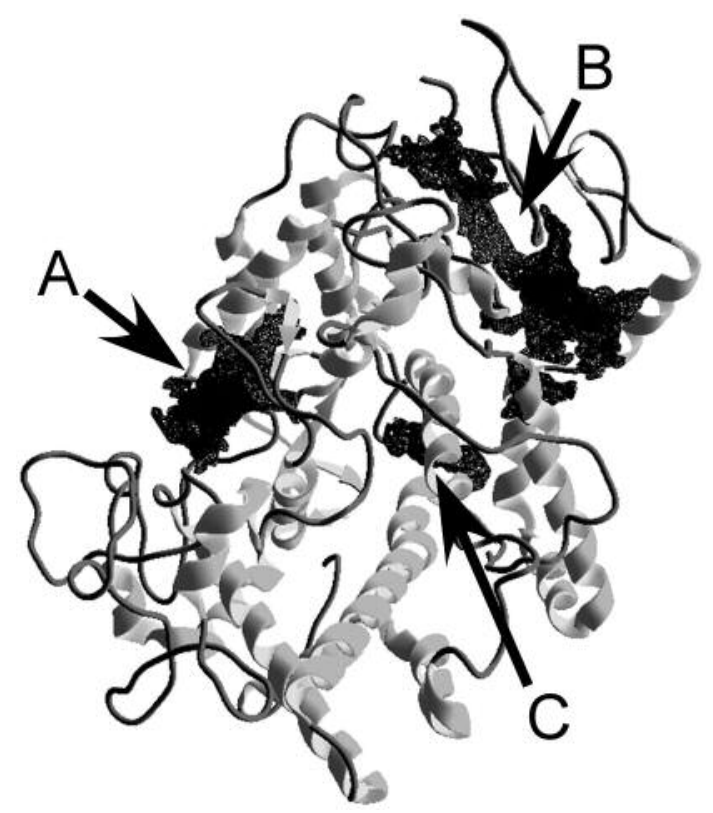

Figure 2. Ligand-binding pockets of cyclo-oxygenase (COX)-2 molecule. Three ligand-binding pockets $A$ to $C$ were determined by molecular simulation.

Table I. Effect of TX series molecules on cyclo-oxygenase (COX)- 1 and COX2 activity.

\begin{tabular}{lccc}
\hline & \multicolumn{3}{c}{$\mathrm{IC}_{50}(\mathrm{M})$} \\
\cline { 2 - 4 } Compound & $\mathrm{COX} 1$ & $\mathrm{COX} 2$ & $\mathrm{R}_{\mathrm{C} 1 / \mathrm{C} 2}$ \\
\hline TX-1123 & $1.57 \times 10^{-5}$ & $1.16 \times 10^{-6}$ & 13.5 \\
TX-1925 & $4.77 \times 10^{-5}$ & $1.03 \times 10^{-5}$ & 4.63 \\
TX-1918 & $7.35 \times 10^{-5}$ & $6.56 \times 10^{-4}$ & 0.11 \\
TX-1926 & $6.84 \times 10^{-6}$ & $8.86 \times 10^{-6}$ & 0.77 \\
TX-1934 & $1.82 \times 10^{-4}$ & $8.15 \times 10^{-4}$ & 0.22 \\
Tyrphostin AG17 & $2.29 \times 10^{-3}$ & $3.23 \times 10^{-4}$ & 7.09 \\
Celecoxib & $2.80 \times 10^{-6}$ & $9.10 \times 10^{-8}$ & 30.8 \\
Indomethacin & $3.10 \times 10^{-6}$ & $1.50 \times 10^{-4}$ & 0.02 \\
\hline
\end{tabular}

$\mathrm{IC}_{50}$ : Half maximal-inhibitory concentration; $\mathrm{R}_{\mathrm{C} 1 / \mathrm{C} 2}$ : inhibition ratio of COX1/COX2 as determined by the ratio of $\mathrm{IC}_{50}$ values for COX1 and COX2.

values $\left[2.80 \times 10^{-6} \mathrm{M}(\mathrm{COX} 1)\right.$ and $\left.9.10 \times 10^{-8} \mathrm{M}(\mathrm{COX} 2)\right]$ were summarized from previous report (13).

Ligand-bindable pockets in COX2. The COX2 molecule flexibly moved during the MM-MD simulation (data not shown). Three major ligand-bindable pockets were observed in the COX2 molecule (Figure 2). Similar ligand-bindable pockets to those in the $\mathrm{COX} 2$ molecule were also detected in the COX1 molecule (data not shown). 


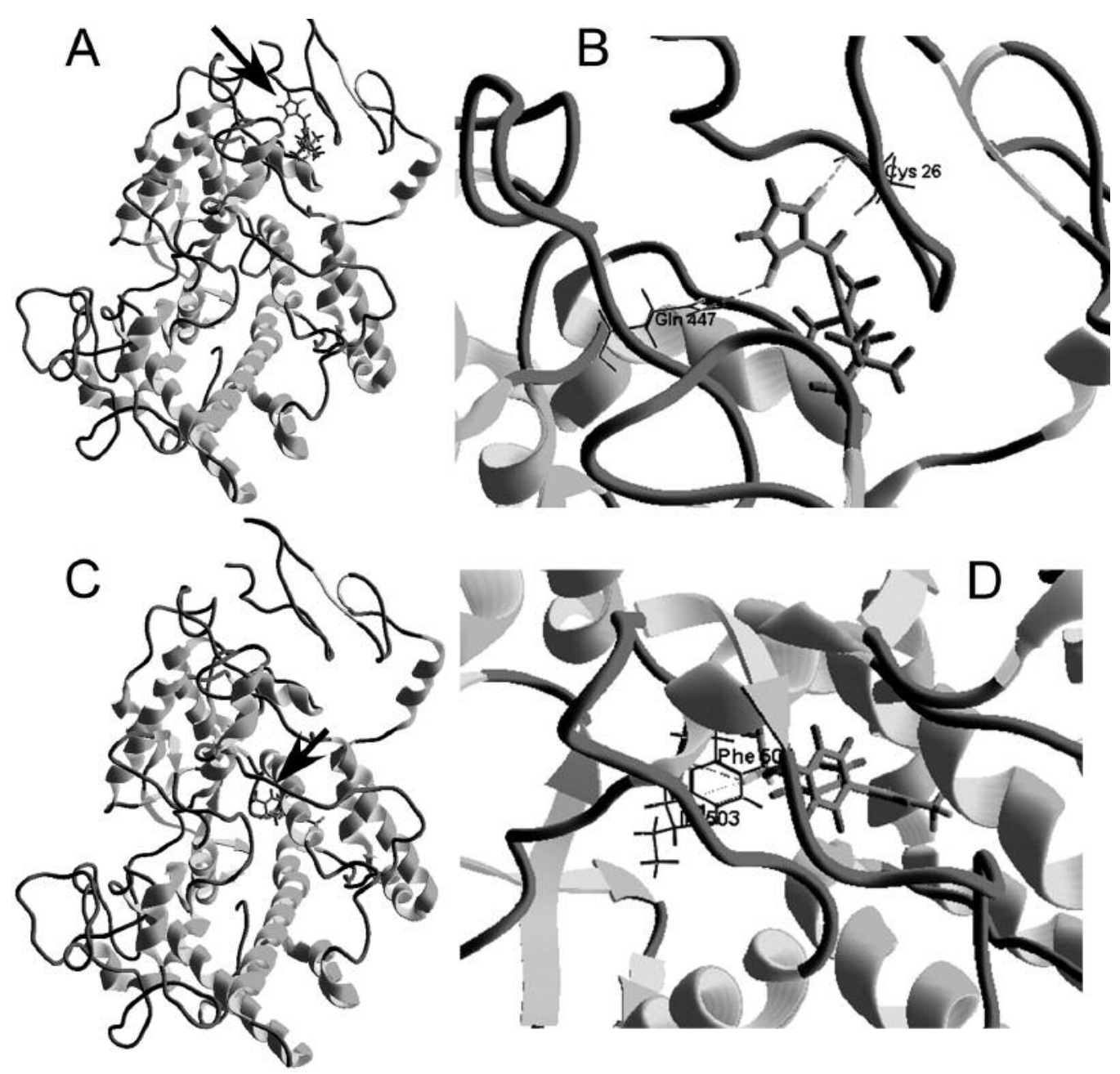

Figure 3. Binding profiles of ligands to cyclo-oxygenase (COX)-2. A: TX-1123 bound to pocket B (Figure 2) of COX2. B: The cyclopentene-1,3dione of TX-1123 interacted with Cys ${ }^{26}$ and Gln ${ }^{447}$ of COX2. C: Celecoxib bound to pocket C of COX2. D: The sulfonamide group of celecoxib interacted with Ile $e^{503}$ and Phe 504 of COX2.

Interaction between $T X-1123$ and COXs. TX-1123 bound at COX2 pocket $\mathrm{B}$ (Figure 2), and the oxygen atom of the 4cyclopentene-1,3-dione region of TX-1123 interacted with $\mathrm{Cys}^{26}$ (nitrogen atom) and $\mathrm{Gln}^{447}$ (the nitrogen atom of the amide group) (Figure 3A and B). COX2-selective celecoxib bound at pocket $C$ (Figure 2), and the oxygen atom of the sulfonamide group interacted with both $\mathrm{Ile}^{503}$ (nitrogen atom) and $\mathrm{Phe}^{504}$ (nitrogen atom) (Figure 3C and D). The nitrogen atom of the sulfonamide group of celecoxib interacted with $\mathrm{Phe}^{504}$ (the oxygen atom of the hydroxy group).

TX-1123 bound COX1 pocket $\mathrm{B}$, and the oxygen atom of the 4-cyclopentene-1,3-dione group of TX-1123 interacted with $\mathrm{Cys}^{41}$ (nitrogen atom) and $\mathrm{Gln}^{461}$ (the nitrogen atom of the amide group) (Figure 4A and B). The oxygen atom of the TX-1123 phenolic group interacted with COX1 Arg ${ }^{469}$ (the nitrogen atom of the side chain). Indomethacin bound to COX1 pocket $\mathrm{C}$ (Figure 4C), and the carboxyl group oxygen atom of indomethacin interacted with COX1 $\mathrm{Tyr}^{355}$ (phenolic hydroxyl group) (Figure 4D). Indomethacin also bound to COX 2 pocket $\mathrm{A}$, and interacted with $\mathrm{COX} 2 \mathrm{His}^{372}$ and $\mathrm{Thr}^{198}$ (data not shown).

Electrostatic potential (ESP) interaction between TX-1123 and COX2. The interactive feature of ESP fields between TX1123 and COX2 is shown in Figure 5. An ESP field was not detected at the position of TX-1123, whereas positive and negative ESP fields were distributed around the TX-1123binding pocket (Figure 5). This distribution pattern of ESP fields was similar to that of the celecoxib-bound COX2 molecule (data not shown). 

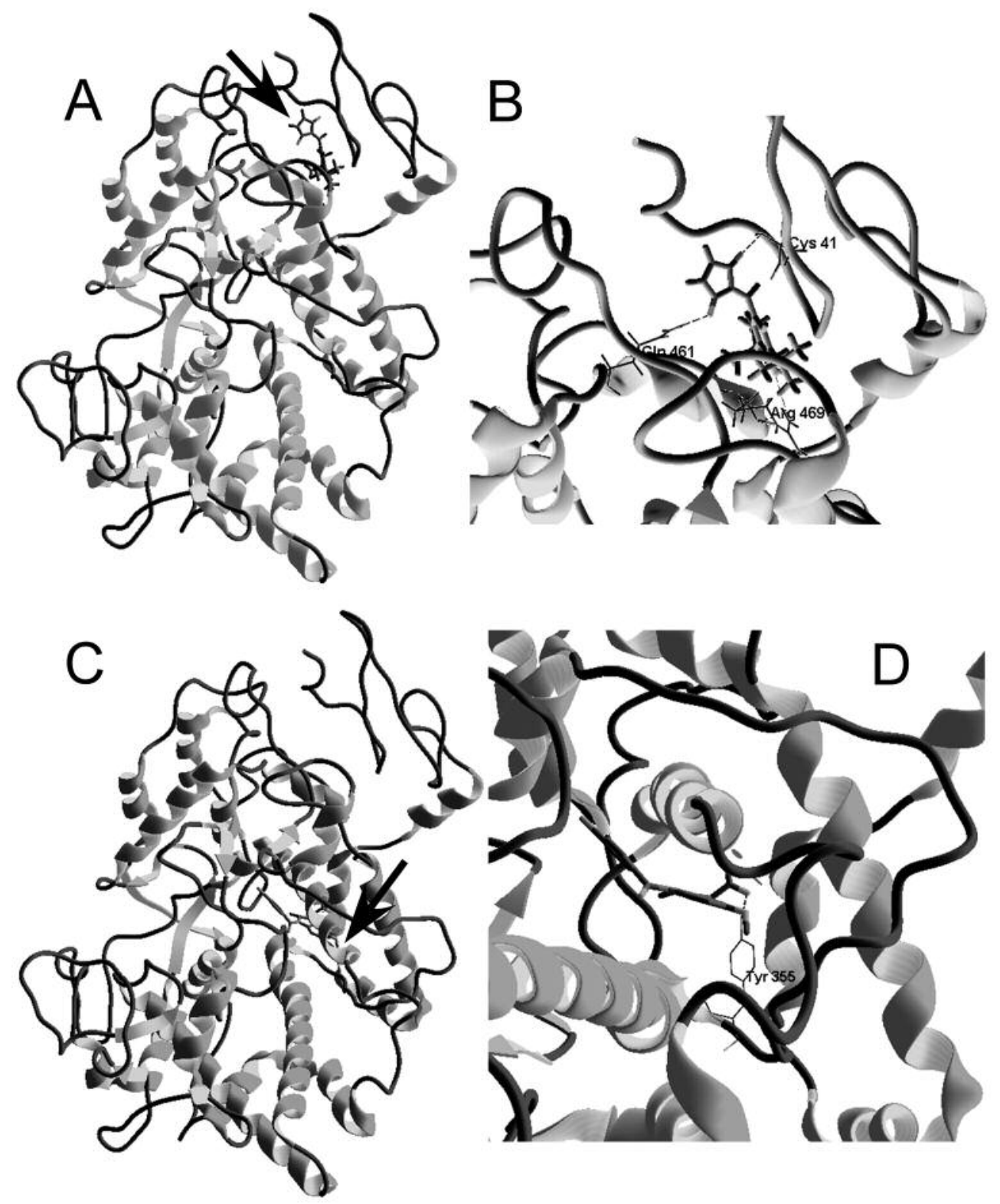

Figure 4. Interaction profiles of ligands to cyclo-oxygenase (COX)-1. A: TX-1123 bound to pocket B of COX1. B: Cyclopentene-1,3-dione of TX1123 interacted with $\mathrm{Cys}^{41}$ and Gln ${ }^{461}$ of COX1. Phenolic group of TX-1123 interacted with Arg 469 of COX1. C, D: Indomethacin bound to pocket $C$ of COX1.

\section{Discussion}

The development of COX2-selective inhibitors was examined, and the molecular design and synthesis of TX-1123 derivatives was performed (10). TX-1123 exhibited potent COX2inhibitory activity, with an $\mathrm{R}_{\mathrm{C} 1 / \mathrm{C} 2}$ value of 13.5 , which was close to that of celecoxib (30.8) (Table I). The $\mathrm{R}_{\mathrm{C} 1 / \mathrm{C} 2}$ value decreased to 4.63 for TX-1925, which possesses a methoxy group instead of the hydroxy group in TX-1123 (Figure 1). The
$\mathrm{R}_{\mathrm{C} 1 / \mathrm{C} 2}$ value of TX-1918, which had two substituted methyl groups at the tert-butyl groups, decreased significantly to 0.11 . The surrounding structure of the phenolic hydroxy group appears to be important for COX2 selectivity. The $\mathrm{R}_{\mathrm{C} 1 / \mathrm{C} 2}$ value was 0.77 for TX-1926, which was introduced at a benzene ring into the cyclopentene-1,3-dione region, and a conjugate system located far from the phenolic hydroxy group in TX-1123 may contribute to COX2 selectivity. It was not possible to confirm the COX2 selectivity of the designed TX-1123 series, except 


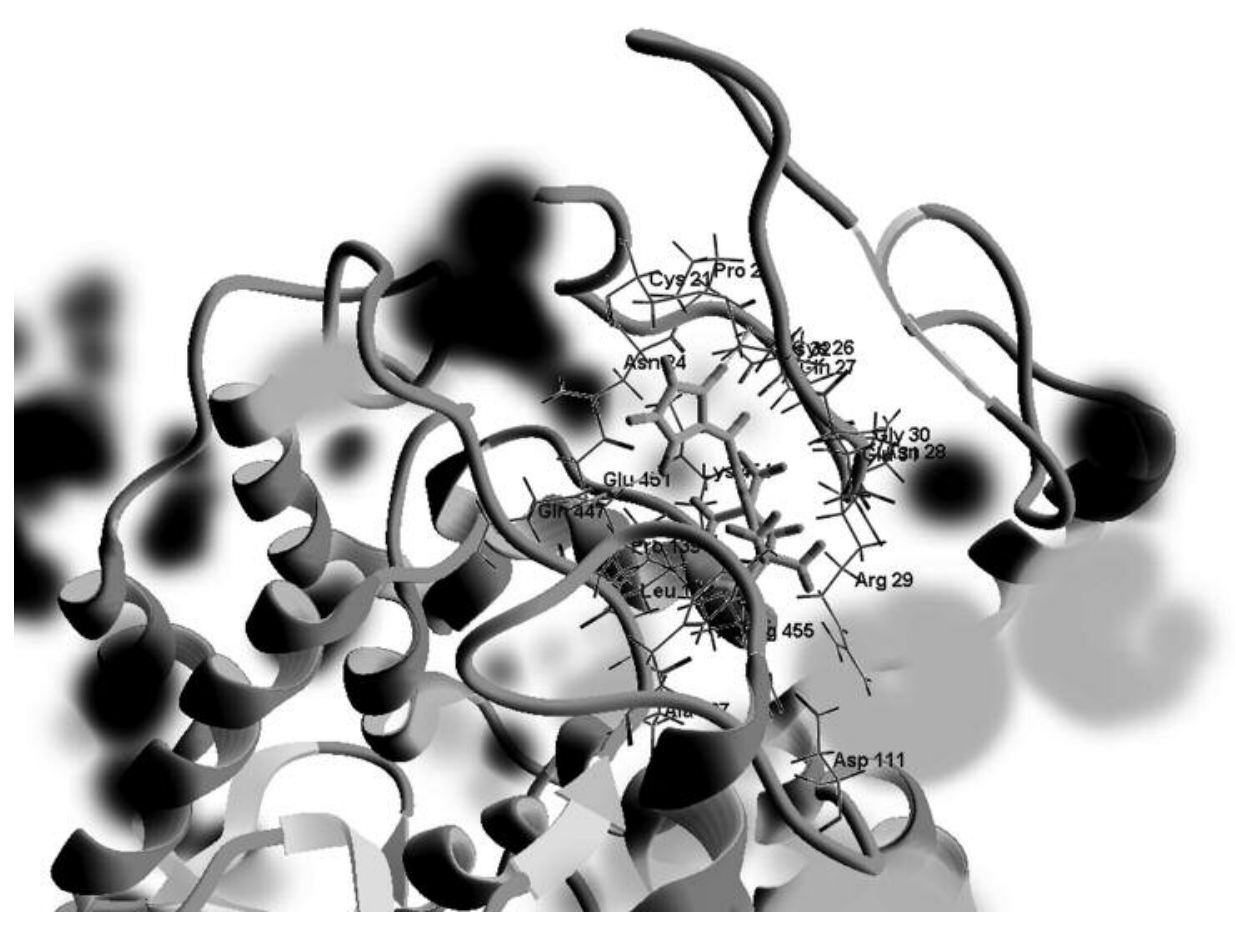

Figure 5. Electrostatic potential (ESP) field profile of TX-1123 binding pocket of cyclo-oxygenase (COX)-2. Positive (gray clouds) and negative (dark gray clouds) ESP fields were distributed around the TX-1123-binding pocket of COX2.

for TX-1123, which appears to be a useful lead structure for the design of more efficient compounds.

Three ligand-bindable pockets were detected in the COX2 molecule, and binding of TX-1123 to pocket B was suggested by the binding simulation (Figure 2). Celecoxib bound to pocket $\mathrm{C}$ of $\mathrm{COX} 2$, which differed from the TX1123-binding pocket (Figure 3 ). The binding mechanism of TX-1123 to COX2 is expected to differ from that of celecoxib. The $\mathrm{IC}_{50}$ value of TX-1123 was higher than that of celecoxib, while the binding affinity of TX-1123 appeared to be weaker than that of celecoxib. TX-1123 was also bindable to COX1 (pocket B), which differed from the indomethacin-binding pocket (pocket $\mathrm{C}$ ) (Figure 4). The COX1-inhibitory activity of TX-1123 was one order weaker than that for COX2. The COX1-inhibitory activity of indomethacin was two orders stronger than its COX2-inhibitory activity, and the COX1-inhibitory mechanism of indomethacin appears to differ from that of TX-1123.

An ESP field did not exist at the TX-1123-bound COX2 pocket, and positive- and negative-ESP fields surrounded the TX-1123-binding pocket (Figure 5). Thus, strong electrostatic energy does not appear to be necessary for the binding reaction between TX-1123 and COX2. A rigid interactive relationship, such as the lock-and-key model of enzyme and substrate, does not appear to be important for the COX2selectivity of TX-1123 family members. Protein-ligand interactions have been examined using isothermal titration calorimetry, and molecular parameters (e.g. enthalpy, entropy) have been determined (16). Enthalpy and entropy are used as components of binding free energy, and these parameters are applied to drug design. An optimized drug which is highly efficient and has few side-effects exhibits more enthalpydominated features (17). A dominant analysis of enthalpy with TX-1123 derivatives is currently in progress in order to obtain a better understanding of the interaction mechanisms between TX-1123 family members and the COX2 molecule.

\section{Acknowledgements}

This work was supported, in part, by Grant-in-Aid for Scientific Research (15K08113) from the Ministry of Education, Culture, Sports, Science and Technology of the Japanese Government. Authors thank Prof. Toshio SATOH (Faculty of Pharmaceutical Sciences, Tokushima Bunri University) for examination of COX activity.

\section{References}

1 Steinmeyer J: Pharmacological basis for the therapy of pain and inflammation with nonsteroidal anti-inflammatory drugs. Arthritis Res 2: 379-385, 2000. 
2 Suleyman H, Demircan B and Karagoz Y: Anti-inflammatory and side-effects of cyclo-oxygenase inhibitors. Pharmacol Rep 59: 247-258, 2007.

3 Baek SJ and Eling TE: Changes in gene expression contribution to cancer prevention by COX inhibitors. Prog Lipid Res 45: 1$16,2006$.

4 Watanabe T, Tanigawa T, Nadatani Y, Otani K, Machida H, Okazaki H, Yamagami K, Watanabe K, Tominaga K, Fijiwara Y and Arakawa T: Mitochondrial disorders in NSAIDs-induced small bowel injury. J Clin Biochem Nutr 48: 117-121, 2011.

5 Wang X, Baek SJ and Eling T: COX inhibitors directly alter gene expression: Role in cancer prevention? Cancer Metastasis Rev 30: 641-657, 2011.

6 Crofford LJ: COX-1 and COX-2 tissue expression: implications and predictions. J Rheumatol 24: 15-19, 1997.

7 Ohkura $\mathrm{K}$ and Hori $\mathrm{H}$ : Modification of cell response to insulin by membrane-acting agents in rat white adipocytes: analysis of structural features by computational simulation. Bioorg Med Chem 9: 3023-3033, 2001.

8 Palumbo G A, Yarom N, Gazit A, Sandalon Z, Baniyash M, Kleinberger-Doron N, Levitzki A and Ben-Yehuda D: The tyrphostin AG17 induces apoptosis and inhibition of CDK2 activity in a lymphoma cell line that overexpresses BCL-2. Cancer Res 57: 2434-2439, 1997.

9 Terada H, Fukui Y, Shinohara Y and Ju-ichi M: Unique action of a modified weakly acidic uncoupler without an acidic group, methylated SF 6847, as an inhibitor of oxidative phosphorylation with no uncoupling activity: possible identity of uncoupler binding protein. Biochim Biophys Acta 933: 193-199, 1988.

10 Hori H, Nagasawa H, Ishibashi M, Uto Y, Hirata A, Saijo K, Ohkura K, Kirk K L and Uehara Y: TX-1123: An antitumor 2hydroxyarylidene-4-cyclopentene-1,3-dione as a protein tyrosine kinase inhibitor having low mitochondrial toxicity. Bioorg Med Chem 10: 3257-3265, 2002.
11 Ohkura K, Kawaguchi Y, Tatematsu Y, Uto Y and Hori H: An antitumor 2-hydroxyarylidene-4-cyclopentene-1,3-dione as a protein tyrosine kinase inhibitor: Interaction between TX-1123 derivatives and Src kinase. Anticancer Res 36: 3645-3649, 2016.

12 Futaki N, Takahashi S, Yokoyama M, Arai I, Higuchi S and Otomo S: NS-398, a new anti-inflammatory agent, selectively inhibits prostaglandin $\mathrm{G} / \mathrm{H}$ synthase/cyclooxygenase (COX-2) activity in vitro. Prostaglandins 47: 55-59, 1994.

13 Yoshino T, Kimoto A, Kobayashi S, Noguchi M, Fukunaga M, Hayashi A, Miyata K and Sasamata M: Pharmacological profile of celecoxib, a specific cyclo-oxygenase-2 inhibitor. Arzneimittelforschung 55: 394-402, 2005.

14 Kawaguchi Y, Tabata A, Nagamune $\mathrm{H}$ and Ohkura K: Profiles of ILY, VLY and Sm-hPAF interaction with human CD59. Anticancer Res 33: 2901-2904, 2013.

15 Ohkura K, Kawaguchi Y, Tabata A, Yamamoto A, Shinohara Y, Nagamune $\mathrm{H}$ and Hori $\mathrm{H}$ : Molecular profiles of cholesterol dependent cytolysin family-derived 11 mer regions. Anticancer Res 32: 2343-2346, 2012.

16 Klebe G: Applying thermodynamic profiling in lead finding and optimization. Nat Rev Drug Discov 14: 95-110, 2015.

17 Freire E: Do enthalpy and entropy distinguish first in class from best in class? Drug Discov Today 13: 869-874, 2008. 